\title{
LAS EXPECTATIVAS SOBRE EL FUTURO EDUCATIVO Y LABORAL DE JÓVENES DE LA ESCUELA SECUNDARIA: ENTRE LO POSIBLE Y LO DESEABLE
}

Agustina CORICA*

\begin{abstract}
RESUMEN
En el marco de los cambios ocurridos en el mercado de trabajo y en el sistema educativo en los últimos años, el objetivo del artículo es indagar en las expectativas sobre el futuro educativo y laboral de los jóvenes estudiantes de la escuela secundaria de distintos sectores sociales en cuatro jurisdicciones de Argentina (Ciudad y Provincia de Buenos Aires, Salta y Neuquén). Para ello, se sistematizaron cuarenta y ocho entrevistas a estudiantes del penúltimo año de la escuela media. Los datos analizados se relevaron en el marco del proyecto de investigación «Intersecciones entre desigualdad y educación media —un análisis de las dinámicas de producción y reproducción de la desigualdad escolar y social- en cuatro jurisdicciones». Entre los principales resultados obtenidos está la existencia de una brecha entre las expectativas y las posibilidades futuras, y la configuración de las representaciones sobre el futuro en torno al contexto geográfico y el apoyo familiar.
\end{abstract}

PALABRAS CLAVE: JÓVENES, EDUCACIÓN, FUTURO

* Argentina, Magíster en Diseño y Gestión de Políticas y Programas Sociales, Candidata a Doctora por la Facultad de Ciencias Sociales, Universidad de Buenos Aires. Investigadora del Programa de Investigaciones de Juventud de la Facultad Latinoamericana de Ciencias Sociales (FLACSO) sede Argentina. E-Mail: acorica@flacso.org.ar. 


\title{
EXPECTATIVAS SOBRE O FUTURO EDUCACIONAL E LABORAL DE JOVENS DA ESCOLA SECUNDÁRIA: ENTRE O POSSÍVEL E O DESEJÁVEL
}

\begin{abstract}
RESUMO
No marco das mudanças ocorridas no mercado de trabalho e no sistema educativo nos últimos anos, o objetivo do artigo é conhecer as expectativas sobre o futuro educacional e laboral de jovens estudantes da escola secundária de diferentes setores sociais em quatro jurisdições da Argentina (Cidade e Província de Buenos Aires, Salta e Neuquén). Para isso, foram feitas quarenta e oito entrevistas com estudantes do penúltimo ano do ensino médio. Os dados analisados se inserem no marco do projeto de pesquisa «Intersecções entre desigualdade e educação média - uma análise das dinâmicas de produção e reprodução da desigualdade escolar e social- em quatro jurisdições». Entre os principais resultados obtidos está a existência de uma lacuna entre as expectativas e as possibilidades futuras, bem como a configuração das representações sobre o futuro quanto ao contexto geográfico e o apoio familiar.
\end{abstract}

PALAVRAS CHAVE: JOVENS, EDUCAÇÃO, FUTURO

\section{FUTURE EXPECTATIONS ON EDUCATION AND THE LABOR OF HIGH SCHOOL YOUTH: BETWEEN THE POSSIBLE AND DESIRABLE}

\begin{abstract}
As part of the changes in the labor market and education system in recent years, this article aims to investigate expectations about future education and employment of young high school students from different social sectors in four jurisdictions of Argentina (city and province of Buenos Aires, Salta and Neuquén). For this purpose, a systematized forty-eight student interviews have been carried out on the junior middle school. The data analyzed were surveyed as part of the research project «Intersections between inequality and high school-an analysis of the dynamics of production and reproduction of social inequality-school in four jurisdictions». Among the main results is the existence of a gap between expectations and future prospects, and the shape of future representations about geographical and family support.
\end{abstract}

KEY WORDS: YOUTH, EDUCATION, FUTURE 


\section{INTRODUCCIÓN}

LOS CAMBIOS SOCIALES, CULTURALES y económicos de las últimas décadas implicaron que la inserción social y laboral de los jóvenes se fuera transformando (Miranda y Corica, 2008). Entre las principales tendencias asociadas a dicha transformación se destaca la ampliación de la escolaridad, la mayor permanencia de los jóvenes en sus hogares de origen (Biggart et al., 2002), la combinación del estudio con el trabajo (Corica, 2010). En este sentido, diversos estudios han destacado los cambios estructurales y subjetivos en el mundo del trabajo, así como la metamorfosis de las denominadas transiciones juveniles (Casal, 2000; Senett, 2000).

En este marco, el objetivo del artículo es indagar en las expectativas sobre el futuro educativo y laboral de los jóvenes estudiantes de la escuela secundaria en cuatro jurisdicciones de Argentina (Ciudad y Provincia de Buenos Aires, Salta y Neuquén). Los cambios ocurridos en el mercado de trabajo y en el sistema educativo, así como las prácticas escolares de cada escuela, operan en la visión de futuro de estos jóvenes. Pero estos no son los únicos factores que delimitan el futuro, sino que existen otros elementos que configuran las representaciones sobre el futuro como, por ejemplo, la familia y el contexto geográfico (Corica, 2010).

A lo largo del texto se realiza un breve recuento de las investigaciones sobre las perspectivas y representaciones de los jóvenes vinculadas a la educación y al mercado laboral, poniendo en evidencia la vivencia diferencial en las experiencias escolares de los jóvenes de distintos grupos sociales y la segmentación de oportunidades laborales.

Luego, se presenta el análisis cualitativo realizado a partir de las entrevistas en profundidad que se realizaron en base al estudio desarrollado en 2005-2007 y que formó parte de mi tesis de maestría. ${ }^{1}$ En este análisis se presta especial atención a los condicionantes futuros y a las elecciones y decisiones que tomarán los jóvenes cuando terminen el secundario. En este sentido, se profundiza en la indagación en cómo piensan la combinación del estudio con el trabajo; cuáles son las ca-

1 La investigación se realizó en el marco del proyecto «Intersecciones entre desigualdad y escuela media. Un análisis de las dinámicas de producción y reproducción de la desigualdad escolar y social en cuatro jurisdicciones», período 2005-2007, dirigido por Inés Dussel. 
rreras que piensan seguir, cómo piensan su futura trayectoria laboral y la trayectoria de vida.

Por último, en esta indagación entre las condiciones y las elecciones, «lo posible y lo deseable» dará cuenta de los factores que influyen en el futuro laboral que imaginan los jóvenes.

\section{LA MIRADA DE LOS JÓVENES ESTUDIANTES SOBRE SU FUTURO}

En cuanto a la perspectiva futura, investigaciones que han indagado las relaciones entre las subjetividades de los jóvenes y el mercado de trabajo (Filmus, Kaplan, Miranda y Moragues, 2001) muestran que los jóvenes tienen percepciones bastante ajustadas de lo que sucede en el mercado de trabajo. Filmus enfatiza una paradoja. En general, los jóvenes perciben que egresan con una baja formación para las demandas del mercado de trabajo, pero a la vez sienten que la escuela es el lugar donde han aprendido lo poco que saben. Sin embargo, hay diferencias entre los sectores sociales; los jóvenes advierten que muchos de los saberes demandados provienen del capital social acumulado por las familias (Jacinto, 2006) y por lo tanto, esto hace que las perspectivas a futuro estén ancladas en las posibilidades que otorga el entorno familiar y social, reproduciéndose la desigualdad de origen.

En referencia a las vivencias de la experiencia escolar, se ha destacado la vigencia de escolaridades de «distinta intensidad» entre los jóvenes de diferentes grupos sociales. Así, mientras la experiencia escolar de los alumnos de sectores socioeconómicos altos es intensa y deja una fuerte marca subjetiva, la experiencia escolar de los jóvenes pobres es de «baja intensidad» y muchas veces no otorga una experiencia subjetiva diferencial (Duschastzky y Corea, 2002; Kessler, 2004). En el contexto del debate sobre la importancia subjetiva que adquiere la escolaridad entre los jóvenes de distintos grupos sociales, los estudios han intentado dar cuenta de la articulación de nuevas y viejas desigualdades en el ámbito escolar (Dussel y Southwell, 2004). Es decir, que a las «viejas» desigualdades de clase del capitalismo industrial se han sumado «nuevas» desigualdades más móviles, flexibles y dinámicas, como aquellas asociadas al género, los aspectos regionales y/o geográficos, las formas y estructuras de ingreso, el acceso a las prestaciones sociales y financieras, de educación, salud, entre otras (Fitoussi y Rosanvallon, 1997).

Los estudios mencionados señalan que las trayectorias de los estudiantes y sus carreras escolares se diversifican en el propio funcio- 
namiento escolar, y que el origen social es el factor con mayor peso en el tipo de trayectoria que realizan. De modo complementario, la institución escolar mediatiza las condiciones materiales de vida junto con el capital cultural de entrada y permiten la producción de circuitos y trayectorias diversificadas (Filmus, 2000; Filmus, Kaplan, Miranda y Moragues, 2001; Jacinto, 2006; Miranda, 2007). Es decir, la desigualdad social existe porque hay circuitos educacionales con terminales formalmente iguales, pero que en la realidad son diversos. La selección meritocrática que efectivamente opera dentro de un mismo circuito educativo no alcanza a controlar los efectos de la selección social que genera la desigualdad entre circuitos.

Transitar por el sistema educativo ya no representa garantía de movilidad social ascendente como lo pensaban los sectores medios. Tampoco garantiza una mejor inserción laboral, pero la educación sí sigue siendo el medio necesario para acceder a un trabajo. Los sectores populares, por otra parte, han valorado tradicionalmente la educación sobre todo en relación con el trabajo. Pero cuando hay pocas posibilidades de empleo, cuando se deteriora el mercado de trabajo y las credenciales educativas se devalúan, la valoración de la educación muchas veces queda solo en el imaginario de estos grupos sociales (Filmus, Kaplan, Miranda y Moragues, 2001). Ahora bien, en contextos de crecimiento económico — como el actual en nuestro país-, nos preguntamos si esta valoración y perspectiva se modifican.

\section{Condicionantes y oportunidades futuras}

En este apartado se analizan las condiciones que visualizan los jóvenes estudiantes como posibles y las oportunidades que visualizan como realizables. La pregunta acerca del futuro nos permitirá acercarnos a la mirada que tienen los estudiantes sobre sus condiciones objetivas y sus expectativas subjetivas. En el contexto social y económico, y en el momento particular de sus vidas, el futuro inmediato se convierte un presente cuando los jóvenes deciden, de alguna u otra manera sobre su futuro, un futuro que es pensar - entre otras cosas - cómo se imaginan la futura etapa de sus vidas.

Como plantea Guy Bajoit, la mirada temporal referida al futuro implica aquello que se espera como posible o aquello que puede ser proyectable sin que necesariamente se tenga certeza de alcanzarlo totalmente (Bajoit, 2000). Estas proyecciones no se dan en el vacío, los estudiantes no están aislados del contexto en el cual desarrollan 
sus expectativas. Los condicionantes sociales influyen en la mirada del futuro. La selección subjetiva del camino a recorrer tendrá mayores o menores posibilidades de ser llevadas a cabo en función de las restricciones que les imponga el contexto objetivo en el cual viven.

Ahora bien, en el contexto actual en el que el tiempo se vuelve volátil y líquido, la visión de futuro se va modificando, volviéndose el futuro muchas veces presente sin muchas posibilidades de proyectar y/o planificar. Antes la planificación de la vida estaba organizada en ciclos estandarizados: i) formación, ii) actividad y iii) jubilación. En los últimos años, este modelo se ha modificado y ha perdido preponderancia fruto de las transformaciones de las estructuras sociales y del conjunto del ciclo de vida (Dávila y Ghiardo, 2008; Wyn, 2008; Filmus, Miranda y Otero, 2004; Biggart, Furlong y Cartmel, 2008). En este marco, estudios cercanos al campo de la sociología han estado replanteando la necesidad de vincular la «condición juvenil» en un nuevo contexto y la «situación social» de los jóvenes. En este sentido, el análisis remite a vincular lo territorial y temporal concreto, es decir, la situación social de los jóvenes, y el espacio y tiempo determinado. En otras palabras, cómo los distintos jóvenes viven y experimentan su condición de jóvenes. De allí se conjugan procesos que vinculan a la noción de juventud con nuevos estándares de organización del ciclo de vida: i) alargamiento o prolongación de la juventud como una fase de vida producto de una mayor permanencia en el sistema educativo; ii) el retraso en una inserción sociolaboral y de conformación de familia propia; y iii) mayor dependencia respecto a sus hogares de orígenes y menor autonomía o emancipación residencial (Dávila y Ghiardo, 2008). Estos procesos son los que enmarcan la mirada de los jóvenes.

A continuación, se analiza la visión de los jóvenes estudiantes bajo análisis y se indaga sobre su contexto y situación social respecto a la inserción laboral y educativa. En este sentido, se describen las percepciones que tienen sobre las condiciones socioeconómicas y sobre las expectativas futuras, en otras palabras las condiciones objetivas y las esperanzas subjetivas, respectivamente (Bourdieu, 2006).

\section{a) Condicionantes económico-sociales}

En los relatos de los jóvenes estudiantes, lo primero que destacan son las diferencias sociales y económicas. Los estudiantes mencionan que los jóvenes de bajos recursos no tendrán las mismas posibilidades que los jóvenes de los sectores medios y altos. Los jóvenes de los estratos 
más altos, por ejemplo, tienen la posibilidad de que sus padres carguen con los gastos y mantenimiento de los años de estudios universitarios. La distinción por lo económico es un dato destacable: los que tienen más oportunidades de progresar son los jóvenes que están en mejor posición económica.

Esto se refleja en investigaciones de los últimos años en donde se señala que en nuestro país la escala salarial se amplió y la segmentación del mercado laboral ensanchó las diferencias socioeconómicas. Estos estudios corroboran que los jóvenes que provienen de hogares de menores recursos tienen 3,5 veces más probabilidades de estar desempleado que los jóvenes de hogares con mayores recursos económicos (Lépore y Schlesser, 2004). Además, los jóvenes pobres que logran terminar la escuela secundaria no siempre alcanzan a mejorar su inserción laboral.

El valor del título es desigual en términos tanto de protección contra el desempleo como en lo que respecta a la calidad de los empleos (Salvia y Tuñon, 2003; Filmus, Kaplan, Miranda y Moragues, 2001). Entre las razones de esta diferenciación, está el rol del capital social y de las redes familiares en el acceso a buenos empleos (Jacinto, 1996). Esto coincide con los relatos de los jóvenes estudiantes que identifican las redes sociales como el medio principal por el cual consiguen sus primeros trabajos. Las redes sociales son el elemento por excelencia de reproducción de las desigualdades sociales (Jacinto, 1996). Redes que están vinculadas con los grupos sociales afines a su ámbito socioeconómico.

Es decir, que los jóvenes de los sectores bajos son los más perjudicados. Estudios realizados en los últimos diez años demuestran que el itinerario laboral de los jóvenes en situación de pobreza es recorrido con escasos grados de libertad (Jacinto, 1991; Mekler, 1991; Gallart, Moreno, Cerruti y Súarez, 1992; Macri y Van Kemenade, 1993; Moreno, Suárez y Binstock, 1994; Jacinto, 1995).

La inserción temprana en ocupaciones precarias, inestables y poco calificadas está en relación con una identidad que se construye sobre la base de otra serie de precariedades: pertenecer a barrios marginales y viviendas precarias. Varias investigaciones mencionan que cuando más restringida es la red de relaciones sociales, menores son las oportunidades del joven, especialmente en contextos desfavorables.

En los relatos de los estudiantes de los sectores bajos surge que «ser hijos de», «tener tal apellido» genera situaciones de desigualdad que no tiene que ver con una desigualdad de conocimiento sino de 
diferencias legitimadas por el contexto situacional. En definitiva, injusticias que resaltan la desigualdad de oportunidades por el solo hecho de ser y/o vivir en ciertos contextos sociales y geográficos. Es decir, que la desigualdad está presente en lo cotidiano de los jóvenes.

En este nuevo contexto, de desigualdad y de incertidumbre, en donde la escuela no garantiza para todos el acceso a trabajos de calidad, la familia estaría ocupando un lugar fundamental respecto a las posibilidades futuras. Los jóvenes estudiantes destacan que la familia es su referente y el sostén de su futuro. Los padres, familiares y conocidos son la fuente principal de acceso a un trabajo.

\section{b) Condicionantes individuales: lo subjetivo}

Además de estos condicionantes socioeconómicos, entre los estudiantes aparece un discurso individual del futuro. Las posibilidades laborales futuras están influidas por los intereses de cada uno. Muchos de los jóvenes entrevistados señalan: «depende de las expectativas que tenga la persona», lo vinculan con las ganas que tenga cada uno para estudiar o hacer algo. Este aspecto fue destacado por los jóvenes de distinto sector social de la escuela a la que asisten. A esto se suma las cualidades personales que generan ventajas o desventajas en la inserción futura. Estas cualidades están vinculadas con: «personas que sepan hablar, que sepan desenvolverse», personas que tengan «buena presencia», que estén «bien vestidos», como ventajas. Es decir, personas más «activas, independientes, prácticas al trabajar», que son las que tienen más personalidad. Los que tengas estas cualidades van a tener más facilidades para conseguir trabajo. En cambio, las personas tímidas van a tener más dificultades, por ejemplo, a estas personas, dicen los estudiantes, «les cuesta aprender», cualidad que la ven como desventaja. Es decir, que las posibilidades que otorga el contexto económica, social y cultural queda enmarcado muchas veces en lo personal e individual (Jacinto, 2004).

\section{c) Condicionantes por el circuito educativo o segmento educativo}

Respecto a los circuitos o segmentos educativos, indagamos sobre las posibilidades que tienen sus compañeros de curso. En esta dirección, aparecen diferentes caminos posibles según la escuela a la que asisten. Es decir, según el segmento educativo no todos los jóvenes estudian- 
tes tendrán las mismas posibilidades y según la escuela no todos los compañeros podrán lograr sus expectativas.

Según el sector social de la escuela a la que asisten las oportunidades se visualizan diferentes. En el caso de los estudiantes de las escuelas de sectores altos, todos los compañeros tendrán oportunidades. No se cuestionan quiénes, sino que hacen referencia a lo que les gustaría hacer, a lo que aspiran a seguir. La distinción pasa por la elección de la carrera o ámbito en donde les gustaría insertarse y no en si tendrán oportunidades laborales futuras.

Por otro lado, los jóvenes estudiantes de las escuelas de los sectores medios refieren en primer lugar a los que quieran progresar, y estos son los que seguirán estudios universitarios. La distinción entre sus compañeros es más individual; es decir, tiene que ver más con el esfuerzo y lo personal que con cuestiones estructurales y económicas. Aunque en algunos casos, surge lo económico condicionando - de cierta manera- el camino a recorrer para obtener mejores oportunidades futuras.

En cambio, en los estudiantes de los sectores bajos las oportunidades futuras son solo para unos pocos. En sus relatos, los jóvenes que asisten a las escuelas de sectores bajos señalan que los compañeros que tienen más posibilidades en el futuro son solo los abanderados, los escoltas, o sea solo los que son muy buenos alumnos. Y también están «los hijos de», los que tienen «apellido». El resto no tiene futuro. Esta situación la viven como injusta y desanima el esfuerzo de continuar estudios superiores. Esto parece reflejar una «ilusión frustrante» en la que la educación no es suficiente para ellos sino para «los elegidos» (Bourdieu y Passeron, 2004).

La visión de los jóvenes de los sectores bajos da cuenta de la desintegración y fragmentación tanto del sistema social como del sistema educativo. Que la mayoría de sus compañeros, a quienes identifican como los que no son buenos alumnos, son los que no seguirán estudiando, son los jóvenes que no tienen futuro, los que muy probablemente caigan en conductas de riesgo (drogadicción, delincuencia, suicidio, etcétera). Es palpar no solamente la devaluación de las credenciales sino la crisis social que están viviendo estos jóvenes.

Como señala Kessler, la deserción escolar erosiona el entramado vincular de los jóvenes, debilitando su integración e interdependencia, y además reduce las oportunidades de empleabilidad futura así como las posibilidades de construcción de un proyecto personal (Kessler, 2004). Sin embargo, entre los estudiantes de sectores bajos aparece esta idea de la valoración de la escuela como herramienta para la vida. 
Como plantea Auyero (1993), para muchos jóvenes de los sectores populares la escuela pasa a tener un valor más allá de la credencial educativa, para ellos la escuela les enseña a que no sean engañados, permitiéndoles resistir a las adversidades de su entorno.

\section{d) Condicionantes geográficos}

La visión local de las posibilidades concretas de conseguir trabajo también fue señalada por los estudiantes. En los relatos, los jóvenes de los sectores sociales altos y medios del interior del país, como son las provincias de Neuquén y Salta, piensan en migrar de sus provincias para seguir estudios superiores ya que consideran que en sus localidades no hay alternativas de educación de prestigio. Además, consideran que tampoco tienen muchas alternativas laborales y que conseguir trabajo se hace más difícil que en otros lugares.

Sin embargo, para otros jóvenes de las escuelas de los sectores bajos, un desarrollo de la provincia puede potenciar las posibilidades de trabajo. En el caso de una localidad del interior de la provincia de Salta y de Neuquén, muchos de los jóvenes estudiantes que cursan en escuelas orientadas al turismo piensan su salida laboral en estos ámbitos. En este sentido, el mercado laboral local abre posibilidades efectivas de inserción para estos jóvenes.

\section{e) Condicionantes de género}

En cuanto a la distinción de género, como señala Jelin, desde la perspectiva de la oferta de empleo persiste una fuerte segmentación ocupacional. Mientras los hombres participan en todo tipo de sector económico, las mujeres urbanas se concentran en los servicios y el comercio, y dentro de ellos desempeñan tareas «típicamente femeninas». Es decir, aquellas definidas socialmente como extensión de las propias de la labor doméstica: para las mujeres populares, el servicio doméstico en otras casas, limpieza y lavado/planchado de ropa, costura, cuidado de niños, ancianos y enfermos; para las mujeres con mayor nivel educativo, secretariado y docencia; esto es, tareas de cuidado y atención personalizada de terceros). Además, frente a situaciones de dificultad económica - a menudo ocasionadas por el desempleo del «jefe de hogar»-, hay mujeres casadas de clase media que no trabajaban y que comienzan a ofrecer una extensión de su labor doméstica para el mercado tales como comidas especializadas, artesanías, etcétera (Jelin, 
2000). En cambio, los hombres se insertan en ocupaciones como ingeniero, arquitecto y abogados, directivos y gerentes en el sector público y privado, así como mecánicos, electricistas, carpinteros y albañiles, trabajos que son típicamente masculinos.

Ahora bien, ¿esta segmentación persiste aún en los jóvenes? Según los relatos de los estudiantes sigue existiendo esta distinción. Las posibles salidas laborales que se imaginan a corto plazo las mujeres jóvenes son trabajos de secretaria/ayudante contable, docente o maestra particular, empleada doméstica. En el caso de los varones jóvenes son profesor de educación física/entrenador, albañil, técnico (mecánico, químico, electromecánico, etcétera), delivery (reparto a domicilio). En términos generales, la distinción de género en el mercado de trabajo no es diferente entre los jóvenes y los adultos, aunque entre los jóvenes aparece la idea de trabajar de lo que surja.

En síntesis, los jóvenes mencionan condicionantes que influyen en las perspectivas sobre su futuro laboral, entre ellos se identificaron aspectos: i) económico-sociales y familiares; ii) educativos; iii) geográficos y iv) subjetivos e individuales (gustos e intereses). Ahora bien, en los relatos de los jóvenes también señalan el peso diferencial que tienen los condicionantes y que, por lo tanto, influyen de manera distinta. Por ejemplo, no es lo mismo ser de los sectores medio o bajo y ser de Salta o Neuquén.

\section{Las elecciones y decisiones sobre el futuro}

En la transición a la vida adulta también toman relevancia las elecciones y decisiones sobre el futuro. Según algunos autores, el presente aparece condicionado por los proyectos o la anticipación del futuro. El tiempo presente no está determinado solamente por las experiencias acumuladas del pasado del sujeto, sino que forman parte de las aspiraciones y los planes futuros (Machado, 2000; Casal, 2002).

Otros autores señalan que en el contexto actual de ausencia de proyectos colectivos, y de escasa perspectiva de ascenso o movilidad social, comienzan a imperar lógicas cada vez más privatizadoras de la vivencia social, que conllevan a los propios sujetos a establecer mundos más privados que públicos y con crecientes niveles de fragmentación social. Sin duda, los jóvenes no escapan a esta realidad y son ellos quienes viven estas incertidumbres y riesgos de quedarse afuera. En el mundo juvenil se han modificado las lógicas de acción, cuestión que ha derivado en que la actual generación joven se imagine trayectorias de vida donde se enfatizan el logro personal por sobre estrategias 
y acciones de tipo colectiva (Sandoval, 2002; Bajoit, 2003). Las percepciones, expectativas y estrategias de construcción de proyectos estarían enmarcadas en esta lógica más individual que social y un futuro condicionado por el presente.

\section{a) Futura trayectoria educativa}

Para avanzar en el análisis de la proyección a futuro de los jóvenes, se describen las distintas visiones de la trayectoria futura. Actualmente, las trayectorias de vida están caracterizadas fuertemente por una individualización y, al mismo tiempo, por una acentuación de los rasgos del riesgo. En este sentido, se habla de «biografía del riesgo», en relación a la necesidad de tomar decisiones en un contexto social caracterizado por una gran incertidumbre (Leccardi, 2005). Pero también se señala que las narrativas biográficas parecen haber perdido su anclaje en la forma de institucionalización de la vida y la dimensión de la continuidad asociada a ella. Para algunos autores, estas narrativas se encuentran fragmentadas en «episodios», cada uno de los cuales tiene su propio pasado y futuro, limitado en alcance y profundidad (Bauman, 1995).

Ahora bien, ¿cómo visualizan los jóvenes su futuro? En términos generales, la vida de los estudiantes del penúltimo año de la escuela secundaria se reparte entre el estudio y el trabajo. A los diecisiete años, estudiar es una de las actividades principales de los jóvenes en Argentina. La tendencia de la expansión de la matrícula en la escuela influye en la permanencia de los jóvenes en el sistema educativo.

Una de las tendencias generales en los últimos años (Filmus, Kaplan, Miranda y Moragues, 2001; Miranda y Otero, 2005; Miranda, Otero y Corica, 2007) es que la mayoría de los estudiantes de la escuela media piensan combinar ambas actividades - estudiar y trabajar-, pero la combinación de estas actividades es pensada de forma muy diferente según el sector social de la escuela a la que asisten. Las posibilidades de mantener ambas actividades van a estar condicionadas muchas veces por lo que quieren y pueden. Asimismo, las posibilidades de continuar estudiando y/o trabajando están vinculadas con el lugar donde viven.

Los jóvenes estudiantes que asisten a las escuelas de sector alto en su mayoría piensan estudiar solamente, sin embargo muchos piensan trabajar antes de finalizar sus estudios universitarios. ${ }^{2}$ Este deseo

2 Estudios recientes señalan que los estudiantes universitarios que piensan trabajar antes de terminar la carrera son mayoritariamente los hijos de 
de combinar estudio y trabajo en este grupo de jóvenes de sectores sociales altos es diferente según la provincia. Para los jóvenes de sectores altos de Salta y de Neuquén, la visión de futuro tiene que ver con conseguir un trabajo para sustentar sus gastos ya que migrar de sus provincias de origen es un paso necesario. La migración está vinculada con la visión de las oportunidades educativas, los estudiantes de estos sectores sociales mencionan que en las jurisdicciones donde viven no hay universidades «de prestigio» y eso significa migrar a otras provincias donde sí encuentran estas posibilidades. La visión de migrar en muchos de estos jóvenes no se cuestiona. Es un «pasaje» necesario para buscar mejores oportunidades futuras (diferencia geográfica). En cambio, migrar no es necesario para seguir estudios superiores entre jóvenes de sectores altos de las otras dos jurisdicciones en estudio (Ciudad de Buenos Aires y La Plata y Conurbano Bonaerense), el trabajo que prioricen va a estar vinculado a sus estudios universitarios para adquirir experiencia laboral.

Otras de las cuestiones que surgen entre los jóvenes de sectores altos es que existe una distinción entre los que piensan en carreras más vinculadas a una salida laboral tradicional y los que piensan seguir una carrera novedosa. Los primeros comparten la visión de una salida laboral rápida y segura o tradicional y los segundos comparten la visión de una salida laboral más complicada y no tan segura o no tradicional y en algunos casos de más corta duración. Estas dos posibilidades también aparecen juntas, hacer una carrera primero y después otra. Esta decisión está condicionada por antecedentes familiares y culturales de lo que sería mejor para un futuro. En este sentido, el mandato social está muy presente en la decisión. Entre estos jóvenes el futuro es pensado en forma dicotómica: están entre lo que les gustaría y lo que les conviene «socialmente», alternativas que no siempre coinciden.

Por ejemplo, a uno de los jóvenes de una escuela de la Provincia de Buenos Aires de sector alto le gustaría seguir periodismo deportivo, pero como es una carrera terciaria piensa seguir una carrera universitaria relacionada con economía, por ejemplo, administración de empresas, carrera de salida laboral rápida y con posibilidades de progreso económico. Dicotomía que expresa una diferenciación de prestigio entre títulos, el certificado universitario por sobre el terciario.

padres con ocupaciones de baja calificación e ingresos, aunque esta tendencia también depende de la carrera y/o área disciplinar (en Riquelme, 2008). 
Otro caso es un joven que piensa seguir estudiando la carrera de arquitectura, pero le gustaría más artes plásticas o diseño gráfico, pero por considerar que no tiene mucha salida laboral optaría por una carrera más tradicional como es arquitectura. Quizá estas dos alternativas tienen más puntos en común, pero siguen siendo alternativas que son diferentes en tanto que la elección está motivada por cuestiones distintas (por el gusto, en un caso, y por lo económico, en el otro). ¿Por qué será que terminan eligiendo este tipo de carrera: porque es una salida laboral rápida, porque tener título universitario tiene prestigio social o es realmente lo que desean?

Ahora bien, la disyuntiva entre seguir una carrera tradicional o no tradicional siempre ha estado presente entre los jóvenes, pero la diferencia es la forma en que hoy en día se resuelve. La elección de carreras tradicionales no les impide seguir simultáneamente carreras alternativas y/o novedosas. Hacer ambas cosas es una forma de pensar la transición más exploratoria que estructuradamente.

Por otro lado, están los jóvenes de los sectores medios y bajos quienes piensan seguir una carrera vinculada con la modalidad de la escuela a la que asisten. En su mayoría son los jóvenes que asisten a escuelas técnicas quienes piensan carreras universitarias vinculadas a las ramas de actividad de la modalidad, por ejemplo, los jóvenes de escuelas técnicas de orientación electrónica piensan seguir ingeniería electrónica. El currículo de las escuelas técnicas está orientado a la formación para el trabajo. En este sentido, la mayoría de los estudiantes que asisten a estas escuelas ven que la modalidad educativa les asegura un futuro laboral. Esto, además, parece una característica diferenciadora de otras modalidades que excede la mera reproducción de las diferencias entre los sectores sociales a los que las escuelas atienden (Corica y Legarralde, 2007). Pero también estudiantes que asisten a escuelas con orientación humanísticas-pedagógicas piensan seguir carreras vinculadas a esta orientación, por ejemplo, psicología o psicopedagogía. Aunque esta orientación no es percibida por los estudiantes como una modalidad que asegura su futuro laboral pero sí orienta la elección de los estudios universitarios.

Por otro lado, algunos jóvenes que asisten a escuelas de sector medio les resulta difícil estudiar solamente. Este grupo de jóvenes piensa que estudiar solamente será posible si consiguen un trabajo para solventar sus gastos. Muchos de estos jóvenes piensan que el futuro se va construyendo, no está dado. Es una visión de futuro en que el esfuerzo personal es el principal elemento. En este grupo apare- 
ce una mirada de la educación como un medio para la movilidad social, como rememorando épocas de sus padres y familiares en donde la carrera laboral era una de las opciones posibles.

En cambio, en los jóvenes de sectores bajos influye mucho más la situación económica familiar; muchas veces por la ausencia de unos de sus progenitores, por problemas económicos o por vivir lejos de una universidad, sostener una carrera universitaria se les hace difícil. Este grupo de jóvenes son los que mencionan la mayoría de las veces en sus relatos la devaluación del título secundario. Ellos señalan la diferente calidad educativa que reciben en sus escuelas, una desigualdad educativa expresada en la desigualdad del valor de su título secundario: «no es igual que el de otros estudiantes que se reciben en otras escuelas, escuelas con mayor prestigio, con calidad educativa».

Esta devaluación puede estar generando entre ellos una visión más pesimista de la mirada del futuro. ¿Se podría pensar que existe una reproducción de las posibilidades futuras? Entre los estudiantes de este sector social muchos de ellos son los primeros en terminar el secundario. La visión de ascenso social a través de la educación es la ilusión de mejorar su situación socioeconómica para este grupo de jóvenes. Pero esta ilusión se une al pesimismo sobre las oportunidades futuras. Más allá de esta sensación, sigue apostando a la escuela como el lugar en donde podrán superar las dificultades y poder «torcer el destino» (Dussel, Brito y Núñez, 2007).

En este grupo de jóvenes es donde con mayor frecuencia se menciona que les resultará más difícil sostener ambas actividades (estudiar y trabajar). Muchos de ellos no podrán terminar estudios superiores por la necesidad de trabajar para subsistir. Esto se vincula a los tipos de trabajos que consiguen mayoritariamente los jóvenes de los sectores bajos: trabajos de muchas horas, mal remunerados, en condiciones muy precarias, lejos de su hogar que implica muchas horas de viaje, y por lo tanto, poco tiempo para el estudio [Salvia (comp.), 2008].

Algunos jóvenes de sectores bajos piensan este tránsito entre la educación y el trabajo como una combinatoria de posibilidades, entre ellas está la posibilidad de trabajar primero, mientras piensan en qué pueden estudiar, realizar una carrera corta, con rápida salida laboral y después, una vez terminada esa carrera, continuar una carrera más larga, vinculada a estudios universitarios. Es decir, no dejan de pensar en continuar estudiando, medio que les dará un futuro mejor, según expresan en sus relatos. 
Otro de los elementos que influyen en la visión de futuro laboral de los jóvenes son los adultos, figura que se destaca con mayor frecuencia entre los estudiantes de los sectores bajos. Los adultos significativos son sus padres y familiares y/o adultos profesionales que tuvieron una presencia importante en sus vidas. Por ejemplo, una joven entrevistada hace referencia a la asistente social que la atendió y la ayudo cuando estuvo en un instituto de menores, otro joven estudiante menciona al psicólogo, otra al médico que fue decisivo en su recuperación de un problema de salud crónico. Estos adultos son referentes para estos jóvenes en la elección de la carrera a seguir.

Entre los estudiantes que asisten a escuelas de sectores bajos, hay poca correlación entre lo que les gustaría y lo que en definitiva se ven haciendo en un futuro. Parecería que las expectativas son «más grandes» que las posibilidades. En muchos de los casos, lo que les gusta estudiar tiene que ver con experiencias personales y con adultos significativos y no con las opciones posibles y efectivas de concretar. Por ejemplo, las dos jóvenes, contrariamente a lo que les gustaría estudiar - medicina y asistente social一, se ven trabajando en la policía o cuidando chicos.

\section{b) Futura trayectoria laboral}

Siguiendo con la vinculación entre las aspiraciones y las expectativas, Bourdieu dice que las aspiraciones tienden a tornarse más realistas, más estrictamente ajustadas a las posibilidades reales, a medida que estas últimas aumentan. La distancia entre el nivel de aspiración y el nivel de realidad, entre las necesidades y los medios, tiende a decrecer a medida que se eleva en el estrato social y, por lo tanto, en el nivel de ingreso. Según Bourdieu, la diferenciación económica va a estar vinculada con las aspiraciones; es decir, que las decisiones futuras se tomarán a partir de las posibilidades efectivas de ser realizadas. Asimismo, el campo de los posibles tiende a ensancharse a medida que uno se eleva en la jerarquía social, solo una minoría de privilegiados se beneficia de un conjunto de seguridades que conciernen al presente y al porvenir. Por lo tanto, el grado de libertad varía considerablemente, las potencialidades objetivas son definidas por el estatuto social y por sus condiciones materiales de existencia (Bourdieu, 2006).

La conciencia de los límites se expresa al mismo tiempo en la esperanza realista de mejorar. Por eso, las aspiraciones tienden a circunscribirse a medida que la posibilidad de satisfacerlas se acrecienta, tal vez porque la conciencia de las dificultades interpuestas se vuelve 
más aguda, como si nada fuese verdaderamente imposible en tanto que nada es verdaderamente posible (Bourdieu, 2006). En este sentido, es que al analizar las respuestas de los jóvenes estudiantes de los sectores altos sobre su trayectoria laboral futura las expectativas y aspiraciones son más realistas y posibles que en los jóvenes de los sectores medios y bajos.

Los jóvenes estudiantes de los sectores medios piensan que tendrán algunas dificultades de trabajar en lo que estudiarían. Sin embargo, esperan poder trabajar de su profesión aunque al principio piensen que tengan que trabajar de lo que les surja. El estrechamiento entre las aspiraciones y la realidad finalmente pueden ajustarse en un camino de lo posible.

En cambio, entre los jóvenes estudiantes de los sectores bajos el futuro laboral es más incierto, la prioridad es trabajar por sobre los estudios superiores y trabajar «de lo que sea». El ajuste entre las aspiraciones y la realidad va a estar condicionado en la mayoría de las veces por su contexto socioeconómico y familiar, y en este estrechamiento y ajuste los caminos pueden ser diversos en cuanto a las diferentes condicionantes.

El estrechamiento entre las aspiraciones y las oportunidades reales entre los estudiantes de los sectores bajos se evidencia en la escasa correlación entre lo que les gustaría y lo que en definitiva se ven realizando en un futuro. En mucho de los casos, lo que les gusta estudiar tiene que ver con experiencias personales y con adultos significativos y no con las opciones que visualizan como posibles y efectivas de concretar. Por otro lado, entre los jóvenes de los sectores medios esta correlación se expresa en distinguir entre lo que les da una salida laboral segura (vinculada con carreras más tradicionales) o salidas laborales más novedosas, como por ejemplo, música, chef, imagen y sonido, etcétera.

\section{c) Futura trayectoria futura}

Como hemos señalando en los apartados anteriores, la visión del futuro queda condicionada por el origen social de los jóvenes estudiantes. Un origen que solo queda a merced de las familias. Para poder asegurar su existencia y crear nuevas formas de vida o adaptarse a los diferentes estándares, los miembros de las familias permanecen juntos mucho más tiempo (la familia como hotel, como soporte), como respuesta a las demandas de la individualización, como una forma de 
amortiguar los riesgos y/o asegurarse ante los mismos (Andreu López Blasco, 2006:263-284).

Si bien es cierto que la familia desempeña un papel central en el apoyo económico de los jóvenes, el vínculo que une a hijos y padres parece pasar a través de dimensiones que no son solamente económicas. Para los jóvenes, la familia representa un escudo contra la incertidumbre, un ancla existencial y emocional capaz de bloquear la ansiedad sobre el futuro. La convivencia prolongada con los padres les facilita la construcción de itinerarios biográficos por ensayo y error, o para iniciar la experimentación existencial, dejando de lado, al menos por el momento, una de las decisiones existenciales de carácter irreversible —entre ellas, conseguir la integración al mundo-. Para los padres, a su vez, continuar con el cuidado de sus hijos es reducir la etapa de la vida conocida como el «nido vacío», que impondría una reestructuración radical de los ritmos diarios y biográficos del tiempo. Por lo tanto, para los padres y los jóvenes, la ampliación de la convivencia se transforma en una cuestión de identidad (Leccardi, 2005).

En la proyección de cómo se piensan en cinco años aparecen los «ritos» de la transición de jóvenes a adultos, ritos que no parecen haber perdido el valor pero sí la forma. La proyección futura de los jóvenes entrevistados fue muy diferente según el sector social de la escuela. En este sentido, los estudiantes de los sectores altos, en su mayoría, se ven terminando la carrera universitaria, viviendo solos e independizándose de su hogar de origen. Es decir, que en este grupo de jóvenes la proyección a futuro es la obtención de la independencia económica y autonomía familiar (Casal, 2000; Biggart et al., 2002; Biggart, Bendir, Cairns, Hein y Morch, 2004; Biggart, Furlong y Cartmel, 2008).

En el caso de los jóvenes de sectores medios, también se ven terminando la carrera universitaria en cinco años, pero en menor medida se ven trabajando con cierta inestabilidad económica personal que les imposibilita independizarse de sus familias. En muy pocos casos se ven viviendo solos. En este grupo de jóvenes, la proyección a futuro en tanto autonomía familiar e independencia económica es más difusa. De hecho en algunos jóvenes de sectores medios les cuesta proyectarse a futuro o dudan lo que podrían estar haciendo. Es como que el futuro es más incierto y que requieren un tiempo mayor que los jóvenes de sectores altos para lograr plena independencia económica y familiar. 
Por último, los estudiantes de las escuelas de sectores bajos se ven en su mayoría trabajando, viviendo en una casa propia o alquilada, habiendo formado su propia familia, algunos se ven con hijos. En este grupo de jóvenes, con mayor frecuencia no se imaginan estudiando o ponen más en duda la posibilidad concreta de terminar sus estudios superiores. La proyección a futuro es plena autonomía familiar e independencia económica, aunque perciben que la estabilidad laboral, y por lo tanto económica, sea más difícil de lograr.

En síntesis, en lo que respecta a la autonomía familiar, los estudiantes de los sectores bajos se acercan a los jóvenes de los sectores altos y en cuanto a la inestabilidad económica se acercan a los de sectores medios. Aunque el logro de ambas cuestiones implique condiciones sociales y económicas muy diferentes entre los mismos jóvenes.

En cuanto a la visión de futuro, es destacable la diferencia en la concepción del «tiempo». El futuro es para algunos un «tiempo» suspendido y para otros un tiempo por planificar. Algunos autores hablan del «tiempo liberado» (Casal, García, Merino y Quesada, 2005), como un tiempo propio de la etapa de la juventud, o sea, propio de su condición juvenil. Esta condición es nueva entre los jóvenes (Bendit, 2005; Wyn, 2008). Es decir, que para los jóvenes de los sectores altos el tiempo liberado aparece como el disfrute, viajar a Europa, vivir experiencias de vida que lo hagan crecer, madurar y conocer realidades distintas.

En cambio, para los estudiantes de los sectores medios este tiempo entre terminar la escuela secundaria y el ingreso al mercado laboral está condicionado por un tiempo de planificación del futuro inmediato. En los jóvenes de los sectores medios, especialmente entre los varones, aparece una visión del tiempo libre como un tiempo fuera de las obligaciones del trabajo y del estudio, un tiempo para disfrutar y para realizar sus pasatiempos. En este sentido, muchas veces se combinan este tiempo «liberado» en el marco de un gusto por una actividad (por ejemplo, la música o el arte) que en algunos casos puede estar vinculada con un futuro laboral. En cambio, en las mujeres jóvenes este tiempo «libre» se lo piensa en la búsqueda de su vocación, es decir, decidir qué estudiar.

En cambio, en los jóvenes de sectores bajos el tiempo liberado no aparece del todo un tiempo de disfrute sino de elecciones acotadas y de un tiempo en suspenso que deberán definir en un corto tiempo. Se podría decir que es un «tiempo libre» que se constituye a través de la frustración y la desdicha en muchos casos (Margulis y Urresti, 1996). En los jóvenes de los sectores bajos, especialmente en los varones, este 
tiempo libre puede ser muchas veces un tiempo del peligro, de caer en conductas de riesgos (delincuencia, drogadicción, suicidio) en vez de ser un tiempo desafiante para experimentar experiencias placenteras. Y en las mujeres de estos sectores sociales, este tiempo es combinado con el trabajo, «mientras busco trabajo y/o trabajo pienso qué carrera seguir» $\mathrm{O}$ «busco un trabajo para sustentarme los estudios».

\section{COMENTARIOS FINALES: EN LO POSIBLE Y LO DESEABLE}

Varios estudios han venido corroborando que el vínculo educación y trabajo se ha modificado (De Ibarrola, 2004; Miranda, 2007; Miranda, Otero y Corica, 2007) y ponen en evidencia la complejidad de este vínculo en nuestros días. Antes, la relación entre la educación y el trabajo era lineal, primero estudiaban y después con el título obtenido salían a buscar trabajo. Ahora cada vez más los estudiantes piensan en combinar el estudio y el trabajo. Pero esta combinación tiene sus particularidades, las formas de combinación dependen de las expectativas a futuro y del sector social al que pertenecen los jóvenes.

Las distintas posibilidades futuras de inserción laboral de los jóvenes entrevistados dejan entrever que la desigualdad educativa tiene una dimensión subjetiva. Los trabajos futuros que visualizan los estudiantes muestran que el título secundario tiene un peso diferencial: a igual certificación obtenida, desigual perspectiva de futuro laboral. A su vez, de la investigación surge que existe un desajuste entre expectativas de inserción laboral que pueda otorgarle el título escolar y las oportunidades objetivas de logro. Estas expectativas se basan en experiencias previas que son la síntesis de la interacción con el mundo cotidiano y funcionan como esquemas de referencia, como hojas de rutas frente al contexto que les toca vivir. Por eso mismo, la forma de pensar la inserción al mundo laboral y social está estrechamente relacionada con las divisiones sociales que organizan esta visión. El conocimiento de las oportunidades futuras lo van construyendo a partir de los intercambios cotidianos - conocimiento, información, experiencias, etcétera-, es un conocimiento socialmente elaborado y compartido con su entorno más próximo. Esto es lo que les da a estos jóvenes el sentido de los límites (Bourdieu, 1998).

En este marco, se ponen en evidencia los fenómenos de individuación y los nuevos aspectos en el análisis de las desigualdades educativas y laborales de los jóvenes. Estos aspectos están relacionados entre otros aspectos con las dinámicas que adquieren las nuevas y 
viejas desigualdades en distintos espacios geográficos, el papel de los grupos familiares y los «adultos significativos» en las trayectorias juveniles, la expansión de «nuevas ocupaciones juveniles» y su impacto en la subjetividad; $y$, el marco regulatorio de las prácticas laborales en distintos espacios jurisdiccionales.

Entre presente y futuro, entre sueños y decisiones, entre lo ideal y lo posible, los jóvenes se van haciendo adultos y ocupando un lugar en la sociedad, configurando su transición y trazando una trayectoria. En la vida de los jóvenes se ponen en juego las experiencias y las expectativas en la definición del futuro. Ambas se entrecruzan internamente, no hay expectativas sin experiencias, no hay experiencias sin expectativas (Koselleck, 1993). Es decir, que «las condiciones de posibilidad de la historia real son, a la vez, las de su conocimiento» expectativas (Koselleck, 1996).

El pasado y el futuro no llegan a coincidir nunca, como tampoco se puede deducir totalmente una expectativa a partir de la experiencia. Pero quien no basa su expectativa en su experiencia también se equivoca. En este sentido, Koselleck habla de «espacio de experiencia» y «horizonte de expectativas». En donde el espacio de experiencia es el lugar donde los acontecimientos del pasado se hacen presente como punto de partida («lo posible») y el horizonte de expectativas delimita la línea tras de la cual se abre en el futuro un nuevo espacio de experiencia («lo deseable»).

Ahora bien, el futuro no tiene una continuidad lineal con el presente como ocurría con las generaciones anteriores sino que puede tener infinitos recorridos y discontinuidades. En el actual contexto, el futuro ya no se presenta con certeza, no se puede programar sino por el contrario es impredecible y volátil. Justamente la incertidumbre del futuro permite pensar en que existan posibilidades diferentes de las actuales, aunque se conozcan las restricciones y límites. Las cosas pueden cambiar, lo que «es así» podría ser modificado por el azaroso tiempo por venir (Saintout, 2006), abriendo una esperanza de que el futuro pueda ser cambiado aunque para algunos jóvenes el presente esté dado y el futuro no sea más que la proyección del presente.

Buenos Aires (ARGENTINA), NOVIEMBRE 2011

RECIBIDO: NOVIEMBRE 2011 ACEPTADO: ABRIL 2012 


\section{REFERENCIAS BIBLIOGRÁFICAS}

BENDIT, RENÉ (2004): «La modernización de la juventud y modelos de políticas de juventud en Europa: análisis comparativos de políticas nacionales en los Estados miembros de la Unión Europea». CINDE, Programa Presidencial Colombia Joven, Centro de Estudios Avanzados en Niñez y Juventud Universidad de Manizales, GTZ y UNICEF (editores): La construcción teórica de la juventud: análisis y perspectivas. Manizales: Gente Nueva.

BIGGART, ANDY et al. (2002): «Trayectorias fallidas, entre estandarización y flexibilidad en Gran Bretaña, Italia y Alemania Occidental». Revista de Estudios de Juventud $\mathrm{N}^{\circ} 56$. Madrid: INJUVE.

— ; A. FURLONG y F. CARTMEL (2008): «Biografía de elección y linealidad transicional: nueva conceptualización de las transiciones de la juventud moderna». En RENÉ BENDIT, MARINA HAHN y ANA MIRANDA (compiladores): Los jóvenes y el futuro: procesos de inclusión social y patrones de vulnerabilidad en el mundo global. Buenos Aires: Prometeo.

—; R. BEndit, D. CAIRnS, K. HeIn y S. MORCH (2004): Families and Transitions in Europe: State of the art report. Ulster, FATE Research Project. School of Social and Community Sciences University of Ulster.

BOURDIEU, PIERRE (2006): Argelia 60: estructuras económicas y estructuras temporales. Buenos Aires: Siglo XXI Editores.

(1998): La distinción: criterio y bases sociales del gusto. Madrid: Taurus.

— y JEAN-ClaUde PASSEON (2004): Los herederos: los estudiantes y la cultura. Buenos Aires: Siglo XXI Editores.

CASAL, JOAQUIM (2000): «Capitalismo informacional, trayectorias sociales de los jóvenes y políticas de juventud. Juventudes y empleos: perspectivas comparadas». Madrid: INJUVE.

; M. GARCÍA; R. MERINO y M. QUESADA (2005): «Aportaciones teóricas y metodológicas a la sociología de la juventud desde la perspectiva de la transición». Barcelona: GRET, Universidad Autónoma de Barcelona.

CoricA, Agustina (2010): «Lo posible y lo deseable. Expectativas laborales de jóvenes de la escuela secundaria». Tesis de Maestría en Diseño y Gestión de Políticas y Programas Sociales. Buenos Aires: FLACSO.

— y MARTín LEgARRALDE (2007): «Desigualdad educativa en la formación para un trabajo: una mirada desde los estudiantes del colegio secundario». Ponencia presentada en el XXVII International Congress LASA 2007. Montreal, Canadá.

DÁvILA, ÓsCAR y FELIPE GHIARDo (2005): «De los herederos a los desheredados. Juventud, capital escolar y trayectorias de vida». Revista Temas Sociológicos $\mathrm{N}^{\mathrm{o}} 11$. Santiago: UCSH.

DE IBARROLA, MARÍA (2004): Paradojas recientes de la educación frente al trabajo y la inserción social. Buenos Aires: RedEtis. 
DuschastzKY, Silvia y CRISTINA COREA (2002): Chicos en banda, los caminos de la subjetividad en el declive de las instituciones. Buenos Aires: Editorial Paidós.

Dussel, I.; A. BRITO y P. NúÑEZ (2007): Más allá de la crisis. Visión de alumnos y profesores de la escuela secundaria argentina. Buenos Aires: Fundación Santillana.

— y MYRIAM SOUTHWELL (2004): «La escuela y la igualdad: renovar la apuesta». El Monitor de la Educación, 5 Época, Año III, N7. Buenos Aires: Ministerio de Educación, Ciencia y Tecnología de la Nación.

FILMUS, DANIEL (2000): Lo que el mercado de trabajo no da, la escuela media no presta. Buenos Aires: EUDEBA.

—- ANA MIRANDA y ANALÍA OTERO (2004): «La construcción de trayectorias laborales entre los egresados de la escuela secundaria». En CLAUDIA JACINTO (coordinadora): ¿Educar para qué trabajo? Discutiendo rumbos en América Latina. Buenos Aires: La Crujia, Ediciones-RedEtis.

— ; - y J. ZELARRAYÁN (2003): «La transición entre la escuela secundaria y el empleo: los recorridos de los jóvenes en el Gran Buenos Aires». Estudios de Trabajo №26. Buenos Aires.

; C. KAPLAN; A. MiRANDA y M. MORAGUES (2001): Cada vez más necesaria, cada vez más insuficiente, la escuela media en épocas de globalización. Buenos Aires: Editorial Santillana.

FItOUSSI, JEAN-PAUl y PIERRE RosAnVALlON (1997): La nueva era de las desigualdades. Buenos Aires: Manantial.

Gallart, M. A.; M. Moreno; M. CerutTi y A. SuÁrez (1992): «Las trabajadoras de villas: familia, educación y trabajo». Cuadernos del CENEP No26. Buenos Aires.

FELIPE GHIARDO y ÓSCAR DÁVILA (2008): Trayectorias sociales juveniles. Ambivalencias y discursos sobre el trabajo. Valparaíso: INJUV y Ediciones CIDPA.

JACINTO, CLAUDIA (2006): La escuela media: reflexiones sobre la agenda de inclusión con calidad. Buenos Aires: Fundación Santillana.

_ (2004): «Ante la polarización de oportunidades laborales de los jóvenes en América Latina. Un análisis de algunas propuestas recientes en la formación para el trabajo». En CLAUDIA JACINTO (coordinadora): ¿Educar para qué trabajo? Discutiendo rumbos en América Latina. Buenos Aires: La Crujia, Ediciones-RedEtis.

(1996): «Desempleo y transición educación-trabajo en jóvenes de bajos niveles educativos. De la problemática actual a la construcción de trayectorias». Revista Dialógica V.1.

(1995): «Formación profesional y empleabilidad de jóvenes de bajos niveles educativos: ¿una articulación posible?». En MARÍA ANTONIA GALLART (compiladora): Formación para el trabajo en el final de siglo: entre la reconversión productiva y la exclusión social. Buenos Aires: OREALC-UNESCO. 
(1991): «Los adolescentes de sectores populares en el conurbano bonaerense: proyectos de vida, educación y trabajo. Un estudio en escuelas de adultos de Buenos Aires». Series Estudios y Documentos 4. La Plata: Dirección General de Escuelas y Cultura, Centro de Información y Documentación.

; M. Wolf; C. BessegA y M. E. LONGO (2005): Jóvenes, precariedad y sentido del trabajo. Buenos Aires.

Jelin, ElizABETH (2000): Pan y afectos: la transformación de las familias. Buenos Aires: Fondo de Cultura Económica.

KeSsler, GABriel (2004): Sociología del delito amateur. Buenos Aires: Paidós.

KosellecK, ReInHART (1996): Futuro pasado. Buenos Aires: Paidós.

— (1993). Futuro pasado: Para una semántica de los tiempos históricos. Buenos Aires: Paidós.

LECCARDI, CARMEN (2005): «Facing uncertainty: temporality and biographies in the new century». Young: Nordic Journal of Young Research, Volumen 13. London.

LÉPORE y SCHELESER (2004): «Desempleo juvenil en Argentina: perfiles y dinámica». Buenos Aires: Mimeo.

LÓPEZ BLASCO, ANDREU (2006): «La familia como respuesta a las demandas de individualización: ambivalencias y contradicciones». Papers No79. Barcelona: Universidad Autónoma de Barcelona.

MACRI, MARIELA y SOLANGE VAN KEMENADE (1993): Estrategias laborales de jóvenes de barrios carenciados. Buenos Aires: Centro Editor de América Latina.

MARGUlis, MARIO y MARCELO URRESTI (1996): La juventud es más que una palabra. Ensayos sobre cultura y juventud. Buenos Aires: Biblos.

MEKLER, Víctor (1991): «Juventud, educación y trabajo en la Argentina: estudio de la situación laboral de los jóvenes de la Gran Ciudad». Maestría en Ciencias Sociales con orientación en Educación. Buenos Aires: FLACSO.

MIRANDA, ANA (2007): La nueva condición joven: educación, desigualdad y empleo. Buenos Aires: Fundación Octubre.

— y ANALÍA OTERO (2005): «Diversidad y desigualdad en los tránsitos de los egresados de la escuela secundaria». Revista Mexicana de Investigación Educativa, Vol. 10 N²5.

; - - y AGUSTINA CORICA (2008): «Las transformaciones en el mercado de trabajo en la Argentina de los últimos 10 años: desigualdad y precariedad entre los jóvenes». Ponencia presentada en el III Congreso de la Asociación Latinoamericana de Población (ALAP) Córdoba, del 24 al 26 de septiembre.

$-;-$ y — (2007): «Tendencias en el tránsito en la educación secundaria y el mundo del trabajo en el Gran Buenos Aires, Neuquén y Salta». $8^{\circ}$ Congreso Nacional de Estudios del Trabajo. Buenos Aires: ASET. 
MorenO, M.; A. SUÁREZ y G. BINSTOCK (1994): «La realidad de jóvenes urbanos pobres: elementos para una política de capacitación». III Seminario de la Red Latinoamericana de Educación y Trabajo CIID-CENEP: La educación y el trabajo frente a los desafíos del siglo XXI. Buenos Aires.

RIQUELME, G. (directora) (2008): Las universidades frente a las demandas sociales y productivas. Estudio y trabajo de estudiantes universitarios: Acceso al empleo, etapas ocupacionales y expectativas sobre la vida profesional. Tomo II. Buenos Aires: Editorial Miño y Dávila.

SAINTOUT, FloRENCIA (2006): «Jóvenes: el futuro llegó hace rato». La Plata: Facultad de Periodismo y Comunicación Social de la Universidad Nacional de La Plata.

SAlviA, Agustín (compilador) (2008): Jóvenes promesas. Trabajo, educación y exclusión social de jóvenes pobres en la Argentina. Buenos Aires: Miño y Dávila.

e IANINA TUÑON (2003): Los jóvenes trabajadores frente a la educación, el desempleo y la inserción social. Buenos Aires: Fundación Friedrich Ebert.

SENETT, RICHARD (2000): La corrosión del carácter: las consecuencias personales del trabajo en el nuevo capitalismo. Barcelona: Anagrama.

WYN, JOHANNA (2008): «Nuevos patrones de la transición de la juventud en la educación en Australia. Los jóvenes y el futuro». En RENÉ BENDIT, MARINA HAHN y ANA MIRANDA (compiladores): Los jóvenes y el futuro: procesos de inclusión social y patrones de vulnerabilidad en el mundo global. Buenos Aires: Prometeo. 